\title{
MOTIVAÇÃO DE USUÁRIOS DE UMA ESTRATÉGIA DE SAÚDE DA FAMÍLIA EM GRUPOS DE SAÚDE
}

\author{
Motivation of users of a Family Health Strategy in health groups \\ Motivación de usuarios de una Estrategia de Salud de la \\ Familia en grupos de salud
}

Artigo Original

\section{RESUMO}

Objetivo: Identificar os motivos de adesão e permanência dos participantes de grupos de Promoção da Saúde. Métodos: Participaram do estudo 27 usuários de uma Estratégia de Saúde da Família (ESF) do município de Santa Maria, Rio Grande do Sul, nos meses de agosto a novembro de 2014. Utilizou-se um questionário adaptado sobre motivação para a prática de atividades em grupo, com as principais variáveis de adesão e permanência, segundo ordem de prioridade. Os dados foram tabulados no programa Excel 2003 e analisados por meio de estatística descritiva simples. Resultados: Os resultados demonstraram que os fatores que motivam os usuários a aderir aos grupos de promoção da saúde na Atenção Básica estão relacionados à melhora da saúde $(n=19,70 \%)$, melhora de desempenho físico $(n=15,56 \%)$, melhora da autoestima $(n=14,52 \%)$, entre outros. Os motivos relacionados à permanência aos grupos, por sua vez, estão relacionados a manter a saúde $(n=18,67 \%)$, receber atenção e incentivo do professor $(n=18,67 \%)$, receber a atenção dos funcionários $(n=15,56 \%)$ e reduzir o nível de estresse $(n=15,56 \%)$. Conclusão: Diante dos dados observados, com relação aos motivos de adesão aos grupos, os indivíduos tendem a buscar melhores condições de saúde e de qualidade de vida. Já o incentivo a um estilo de vida mais saudável, como também o apoio dos profissionais e demais pessoas envolvidas nas atividades, implica em maior permanência dos usuários no grupo.

Descritores: Promoção da Saúde; Atenção Básica; Educação em Saúde.

\section{ABSTRACT}

Objective: To identify reasons for adhesion and permanence in health promotion groups among participants. Methods: The study included 27 users of the Estratégia de Saúde da Familia - ESF (Family Health Strategy) in the municipality of Santa Maria, Rio Grande do Sul, from August to November 2014. An adapted questionnaire about motivation for group activities was used, with the main adhesion and permanence variables distributed in order of priority. Data were tabulated in MS Excel 2003 and analyzed using simple descriptive statistics. Results: The results showed that the factors that motivate users to join health promotion groups in Primary Care are related to health improvement $(n=19,70 \%)$, physical performance improvement $(n=15,56 \%)$, self-esteem improvement $(n=14,52 \%)$, and others. The reasons related to the permanence in the groups are related to health maintenance $(n=18,67 \%)$, attention and encouragement received from the teacher $(n=18,67 \%)$, attention received from the staff $(n=15,56 \%)$ and stress reduction $(n=15,56 \%)$. Conclusion: According to the data on the reasons for adhesion and permanence in the groups, individuals tend to seek better health and quality of life. In addition, encouragement for a healthier lifestyle and also the support from professionals and other people involved in the activities imply greater permanence of users in the group.

Descriptors: Health Promotion; Primary Care; Health Education.

Recebido em: 29/09/2015 Revisado em: 09/10/2015 Aceito em: 10/12/2015 


\section{RESUMEN}

Objetivo: Identificar los motivos para la adhesión y permanencia de los participantes de grupos de Promoción de la Salud. Métodos: Participaron del estudio 27 usuarios de una Estrategia de Salud de la Familia (ESF) del municipio de Santa María, Rio Grande do Sul, entre agosto y noviembre de 2014. Se utilizó un cuestionario adaptado sobre la motivación de la práctica de actividades de grupo con las principales variables de adhesión $y$ permanencia según el orden de prioridad. Los datos fueron tabulados en el programa Excel 2003 y analizados a través de estadistica descriptiva simple. Resultados: Los resultados demostraron que los factores de motivación de los usuarios para la adhesión a los grupos de promoción de la salud en la Atención Básica se relacionan con la mejora de la salud ( $n=19,70 \%)$, del desempeño físico $(n=15,56 \%)$, de la autoestima $(n=14,52 \%)$, entre otros. Los motivos relacionados con la permanencia en los grupos, por su vez, se relacionan con el mantenimiento de la salud $(n=18,67 \%)$, la atención e incentivo del profesor $(n=18,67 \%)$, la atención de los empleados $(n=15,56 \%)$ y la reducción del nivel de estrese ( $n=15,56 \%)$. Conclusión: Delante los datos observados y respecto a los motivos de la adhesión a los grupos, los individuos parecen buscar mejores condiciones de salud y calidad de vida. El incentivo al estilo de vida más saludable y apoyo de los profesionales y demás personas involucradas en las actividades implica en mayor permanencia de los usuarios en el grupo.

Descriptores: Promoción de la Salud; Atención Primaria de Salud; Educación en Salud.

\section{INTRODUÇÃO}

O envelhecimento populacional é um fenômeno natural, irreversível e mundial. Segundo projeções estatísticas da Organização Mundial de Saúde (OMS), no período de 1950 a 2025, o grupo de idosos no país deverá ter aumentado em quinze vezes, enquanto a população total terá aumentado em cinco vezes. Assim, o Brasil ocupará o sexto lugar quanto ao contingente de idosos, alcançando em 2025 cerca de 32 milhões de pessoas com 60 anos ou mais ${ }^{(1,2)}$.

Desde a década de 1960, observam-se os processos de transição demográfica, epidemiológica e nutricional no país, que resultam em alterações nos padrões de ocorrência das enfermidades. A transição epidemiológica caracterizase pela mudança do perfil de morbidade e de mortalidade de uma população, com diminuição progressiva das mortes por doenças infectocontagiosas e elevação das mortes por doenças crônicas não transmissíveis ${ }^{(1,2)}$.

Entende-se, portanto, que a Promoção da Saúde é uma estratégia de articulação transversal, na qual se confere visibilidade aos fatores que colocam a saúde da população em risco e às diferenças entre necessidades, territórios e culturas presentes no nosso país. Isso acaba demandando a criação de mecanismos que reduzam as situações de vulnerabilidade, defendam radicalmente a equidade e incorporem a participação e o controle social na gestão das políticas públicas $^{(3,4)}$.

Uma das estratégias de implementação da Política Nacional de Promoção da Saúde é o estímulo à inserção de ações de Promoção da Saúde em todos os níveis de atenção, com ênfase na Atenção Básica, voltadas às ações de cuidado com o corpo e a saúde; alimentação saudável e controle do tabagismo ${ }^{(3)}$. Para tanto, as práticas na Atenção Básica devem estimular a participação dos usuários, como forma de ampliar sua autonomia e capacidade na construção do cuidado à sua saúde. Posicionamento este em prol de um enfrentamento positivo dos determinantes e condicionantes do processo saúde-doença, na organização dos serviços de saúde, a partir de lógicas centradas no usuário e no exercício do controle social ${ }^{(5)}$.

Em outras palavras, as ações de promoção à saúde na Atenção Básica consistem em estratégias de prevenção de doenças crônicas não transmissíveis, estímulo a hábitos saudáveis e o incentivo dos usuários ao aumento significativo de sua autonomia e maior zelo em relação à saúde. Dessa maneira, alguns autores afirmam que as ações educativas em saúde podem capacitar indivíduos e grupos na construção de novos conhecimentos, conduzindo a uma prática consciente de comportamentos de promoção da saúde e prevenção de doenças. Essas ações geram novas alternativas de controle das doenças, reabilitação e tomada de decisões que favoreçam uma vida saudável $\mathrm{l}^{(6,7) \text {. }}$

Nesse sentido, a proposta educativa do trabalho em grupo consiste em propiciar um espaço de participação em que todos aprendam e ensinem, reformulem concepções e produzam novos conhecimentos. Além disso, as dinâmicas grupais favorecem a expressão dos participantes, permitindo a valorização de suas experiências de vida e o diálogo com o saber técnico-científico, na busca comum de um envelhecimento com mais autonomia e dignidade ${ }^{(4,8)}$.

Entende-se que esses pressupostos consistem em concepções já incorporadas às políticas de saúde e na prática dos profissionais de saúde. Não obstante, o que leva os usuários dos serviços de saúde da Atenção Básica a participar e a permanecer em atividades dessa natureza? Sob essa perspectiva, este estudo teve como objetivo identificar os motivos de adesão e permanência dos participantes de grupos de Promoção da Saúde.

\section{MÉTODOS}

A presente pesquisa caracterizou-se como de campo, quantitativa, descritiva e exploratória. Consistiu-se em pesquisa de campo por buscar a inserção na realidade da população ou do problema estudado para alcançar as 
respostas ou relações entre as variáveis; e em quantitativa, exploratória e descritiva em função de explorar o cenário de estudo descrevendo as principais variáveis encontradas que interferem no fenômeno estudado ${ }^{(9-11)}$.

Este estudo foi desenvolvido a partir de um projeto com pesquisa de campo (projeto guarda-chuva) realizado através do Programa de Residência Multiprofissional Integrada em Sistema Público de Saúde, com área de concentração na Atenção Básica/Estratégia Saúde da Família (ESF), da Universidade Federal de Santa Maria (UFSM). A intervenção envolveu a participação e realização de atividades em grupos de promoção de saúde em área adstrita a uma ESF.

A intervenção teve como pressuposto promover a educação em saúde a partir de temas de interesse dos usuários participantes e dos profissionais de saúde. Os encontros ocorreram nos meses de agosto a novembro de 2014, junto a dois grupos de promoção da saúde vinculados ao Programa Hiperdia, da Unidade de Estratégia de Saúde da Família Lídia, na cidade de Santa Maria, Rio Grande do Sul. Os grupos participantes desses encontros, mensais, participaram de quatro momentos do estudo. Essas intervenções faziam parte do projeto guarda-chuva de educação em saúde, com abordagem de oito temáticas de interesse dos participantes.

Por entender-se que a opinião e o interesse dos usuários são importantes para sua adesão e permanência nos grupos, aplicou-se um questionário adaptado sobre motivação para a prática de atividades em grupo, organizado pelo Laboratório de Estudos em Práticas Esportivas e Lazer (LAPEL) da Escola Superior de Educação Física da Universidade do Pernambuco ${ }^{(12)}$. Ele continha um rol das principais variáveis de adesão e permanência na atividade, segundo ordem de prioridade, e foi devidamente testado e validado por três professores especialistas e vinculados à Universidade do Pernambuco $^{(12)}$.

Participaram da pesquisa usuários dos grupos de saúde adscritos à referida ESF, com mais de 18 anos de idade e que respondessem ao instrumento de pesquisa. Os critérios de exclusão foram usuários com dificuldade de compreensão e os que deixaram os questionários incompletos. O estudo iniciou com 31 participantes, mas 4 foram excluídos por deixarem a maioria das perguntas em branco. Assim, a amostra comportou 27 pessoas.

Nesse questionário organizado pelo LAPEL, a escala de frequência dos praticantes é trabalhada em duas dimensões. Na primeira, observam-se as frequências dos sujeitos sobre os motivos de adesão nos grupos, segundo os dezoito itens a serem respondidos, numa escala Likert de 4 pontos, na seguinte correspondência: 1 - sem importância, 2 - pouco importante, 3 - importante, e 4 - importantíssimo. Consideraram-se as dimensões relacionadas à saúde e à qualidade de vida, à prática de exercícios físicos, aos aspectos psicossociais, ao bem-estar, ao professor, ao uso do tempo e à influência da mídia. Na segunda, investigamse as motivações dos praticantes, segundo os motivos de permanência nos grupos, sendo contemplados 24 itens sobre a prática do exercício. Nessa dimensão, levou-se em conta saúde, bem-estar, ambiente da prática, aspectos psicossociais, qualidade do corpo docente e de funcionários, e valores estéticos ${ }^{(12)}$.

A análise das respostas dos instrumentos de pesquisa foi apresentada em números percentuais, de acordo com a frequência dos motivos de adesão e permanência

Após as categorias serem definidas e nomeadas, procedeu-se a fusão das categorias analíticas e, por último, foram quantificadas e representadas sob a forma de tabelas. Assim, o método de análise apresentado permitiu uma aproximação ao conteúdo e à estrutura própria da investigação descritiva, associada às técnicas quantitativas de análises. Os dados foram tabulados no programa Excel 2003 e analisados por meio de estatística descritiva simples, sendo os resultados expressos em percentuais, com média e desvio padrão.

A pesquisa seguiu os critérios da ética em pesquisa com seres humanos, sendo aprovada pelo Comitê de Ética da Universidade Federal de Santa Maria (UFSM), sob parecer $\mathrm{n}^{\circ}$. 725.276. Os pesquisadores assinaram o Termo de Confidencialidade, garantindo o sigilo e anonimato dos dados coletados.

\section{RESULTADOS}

Dos 27 participantes do estudo, 23 eram mulheres, perfazendo $85 \%$ do total, e 4 eram homens, o equivalente a $15 \%$ da amostra coletada, com idade média de $55( \pm 11)$ anos.

Convém destacar que os motivos para a adesão e permanência dos participantes dos grupos de saúde receberam análise sob duas perspectivas: 1) o considerado importantíssimo para a maioria dos participantes; 2) a classificada como sem importância pelos mesmos ${ }^{(12)}$.

De acordo com o analisado, destacam-se os diferentes motivos para a adesão dos participantes aos grupos de saúde, sendo os dados ilustrados na Tabela I.

As frequências que mais se destacaram para adesão aos grupos de saúde na categoria importantíssimo estão relacionadas a: melhorar a saúde (70\%); melhorar desempenho físico (56\%); melhorar a autoestima (52\%); professor (52\%); confiar no professor (52\%); relaxar (48\%); recuperar lesões (44\%); praticar exercícios físicos (44\%); gostar de desafios (44\%); reduzir o nível de estresse (44\%); adotar um estilo de vida saudável (41\%); e seguir orientação 
médica (41\%). Já as frequências mais apresentadas na segunda perspectiva, como sem importância, encontram-se relacionadas à influência da mídia, merecendo destaque as propagandas (44\%) e outros programas televisivos (41\%).

O observado como importantíssimo entre os motivos de permanência dos participantes nos grupos de saúde está ilustrado na Tabela II.

As frequências mais apresentadas como importantíssimo são as relacionadas a: manter a saúde (67\%); receber atenção e incentivo do professor (67\%) e receber a atenção dos funcionários $(56 \%)$; reduzir o nível de estresse (56\%); sentir a alegria do ambiente (52\%); prazer no exercício físico $(48 \%)$; melhorar a postura $(48 \%)$; bemestar corporal (48\%); bem-estar no ambiente (48\%); gostar do local (48\%); construir amizades (48\%); ficar mais forte (44\%); ter fácil acesso (44\%); receber incentivos (44\%); emagrecer (44\%); parecer mais jovem (44\%); sentirse realizado (41\%); manter-se em forma; e melhorar a aparência (41\%).

Já as frequências mais apresentadas na segunda perspectiva, como sem importância, são as relacionadas a ter status social $(11 \%)$.

Tabela I - Frequência dos participantes segundo os motivos de adesão. Universidade Federal de Santa Maria, RS, 2014.

\begin{tabular}{|c|c|c|c|c|c|c|c|c|}
\hline \multirow[t]{2}{*}{ Categorias dos motivos } & \multicolumn{2}{|c|}{$\begin{array}{c}\text { Sem } \\
\text { importância }\end{array}$} & \multicolumn{2}{|c|}{$\begin{array}{c}\text { Pouco } \\
\text { importante }\end{array}$} & \multicolumn{2}{|c|}{ Importante } & \multicolumn{2}{|c|}{ Importantíssimo } \\
\hline & f & $\%$ & f & $\%$ & f & $\%$ & f & $\%$ \\
\hline \multicolumn{9}{|l|}{ Saúde e qualidade de vida } \\
\hline Adotar um estilo de vida saudável & - & - & - & - & 16 & 59 & 11 & 41 \\
\hline Melhorar o desempenho físico & - & - & - & - & 12 & 44 & 15 & 56 \\
\hline Auxiliar na recuperação das lesões & - & - & - & - & 15 & 56 & 12 & 44 \\
\hline Melhorar a saúde & - & - & - & - & 8 & 30 & 19 & 70 \\
\hline Seguir orientação médica & - & - & - & - & 15 & 56 & 11 & 41 \\
\hline \multicolumn{9}{|l|}{ Exercícios físicos } \\
\hline Praticar exercícios físicos (hábito) & - & - & 1 & 4 & 14 & 52 & 12 & 44 \\
\hline \multicolumn{9}{|l|}{ Aspectos psicossociais } \\
\hline Melhorar autoimagem & - & - & 1 & 4 & 15 & 55 & 11 & 41 \\
\hline Melhorar autoestima & - & - & - & - & 13 & 48 & 14 & 52 \\
\hline Estar com os colegas & - & - & 1 & 4 & 16 & 59 & 10 & 37 \\
\hline Gostar de desafios & - & - & 4 & 15 & 11 & 41 & 12 & 44 \\
\hline Ser influenciado por amigos & 4 & 15 & 3 & 11 & 11 & 41 & 8 & 30 \\
\hline Sair de casa & - & - & - & - & 18 & 67 & 7 & 26 \\
\hline \multicolumn{9}{|l|}{ Bem-estar } \\
\hline Relaxar & - & - & - & - & 14 & 52 & 13 & 48 \\
\hline Reduzir o nível de estresse & - & - & - & - & 15 & 56 & 12 & 44 \\
\hline Professor & - & - & - & - & 13 & 48 & 14 & 52 \\
\hline Confiar no professor & - & - & - & - & 13 & 48 & 14 & 52 \\
\hline \multicolumn{9}{|l|}{ Tempo } \\
\hline Disponibilizar tempo para prática & - & - & 1 & 4 & 17 & 63 & 9 & 33 \\
\hline \multicolumn{9}{|l|}{ Influência da mídia } \\
\hline Televisão & 11 & 41 & 6 & 22 & 6 & 22 & 2 & 7 \\
\hline Propagandas & 12 & 44 & 7 & 26 & 4 & 15 & 2 & 7 \\
\hline
\end{tabular}

f: frequência de respostas dos participantes. \%: porcentagem das respostas dos pesquisados. 
Tabela II - Frequência dos participantes segundo os motivos de permanência. Universidade Federal de Santa Maria, RS, 2014.

\begin{tabular}{|c|c|c|c|c|c|c|c|c|}
\hline \multirow[t]{2}{*}{ Categorias dos motivos } & \multicolumn{2}{|c|}{$\begin{array}{c}\text { Sem } \\
\text { importância }\end{array}$} & \multicolumn{2}{|c|}{$\begin{array}{c}\text { Pouco } \\
\text { importante }\end{array}$} & \multicolumn{2}{|c|}{ Importante } & \multicolumn{2}{|c|}{ Importantíssimo } \\
\hline & f & $\%$ & f & $\%$ & f & $\%$ & f & $\%$ \\
\hline \multicolumn{9}{|l|}{ Exercício } \\
\hline Sentir prazer na prática & - & - & 2 & 7 & 12 & 44 & 13 & 48 \\
\hline Sentir bem-estar & - & - & - & - & 18 & 67 & 9 & 33 \\
\hline \multicolumn{9}{|l|}{ Saúde } \\
\hline Ficar mais forte & - & - & 3 & 11 & 12 & 44 & 12 & 44 \\
\hline Melhorar a postura & - & - & - & - & 14 & 52 & 13 & 48 \\
\hline Manter a saúde & - & - & - & - & 9 & 33 & 18 & 67 \\
\hline \multicolumn{9}{|l|}{ Bem-estar } \\
\hline Aumentar o bem-estar corporal & - & - & - & - & 14 & 52 & 13 & 48 \\
\hline Recuperar-se das atividades diárias & - & - & 2 & 7 & 15 & 56 & 9 & 33 \\
\hline Reduzir o nível de estresse & - & - & 2 & 7 & 10 & 37 & 15 & 56 \\
\hline \multicolumn{9}{|l|}{ Ambiente } \\
\hline Sentir bem-estar & - & - & - & - & 14 & 52 & 13 & 48 \\
\hline Gostar do local da prática & - & - & 1 & 4 & 13 & 48 & 13 & 48 \\
\hline Sentir alegria do ambiente & - & - & - & - & 13 & 48 & 14 & 52 \\
\hline Ter fácil acesso & - & - & 1 & 4 & 14 & 52 & 12 & 44 \\
\hline \multicolumn{9}{|l|}{ Psicossociais } \\
\hline Sentir-se realizado & - & - & - & - & 15 & 56 & 11 & 41 \\
\hline Construir amizades & 1 & 4 & - & - & 13 & 48 & 13 & 48 \\
\hline Ter status social & 3 & 11 & 4 & 15 & 14 & 52 & 6 & 22 \\
\hline Aumentar contato social & 1 & 4 & 2 & 7 & 15 & 56 & 9 & 33 \\
\hline Receber incentivos & - & - & 3 & 11 & 12 & 44 & 12 & 44 \\
\hline \multicolumn{9}{|l|}{ Profissionais do local da prática } \\
\hline Receber atenção do professor & - & - & - & - & 8 & 30 & 18 & 67 \\
\hline Receber incentivos do professor & - & - & 1 & 4 & 9 & 33 & 18 & 67 \\
\hline Receber atenção dos funcionários & - & - & - & - & 11 & 41 & 15 & 56 \\
\hline \multicolumn{9}{|l|}{ Valores estéticos } \\
\hline Manter-se em forma & - & - & - & - & 16 & 59 & 11 & 41 \\
\hline Emagrecer & - & - & - & - & 15 & 56 & 12 & 44 \\
\hline Melhorar a aparência & - & - & - & - & 16 & 59 & 11 & 41 \\
\hline Parecer mais jovem & - & - & 2 & 7 & 13 & 48 & 12 & 44 \\
\hline
\end{tabular}

f: frequência de respostas dos pesquisados. \%: porcentagem das respostas dos pesquisados.

\section{DISCUSSÃO}

O estudo realizou-se com 27 participantes, com idade média de $55( \pm 11)$ anos, dos quais $85 \%$ eram do gênero feminino. Quanto à idade, vale chamar atenção que existem muitos estudos com ações direcionadas a idosos; nesta pesquisa, todavia, verificou-se uma população adulta com menos de 60 anos, demonstrando que indivíduos mais jovens estão em busca de orientação e cuidado para uma vida mais saudável. Um estudo realizado em Pelotas/RS, pelo Núcleo de Atividades para a Terceira Idade (NATI), da
Universidade Federal de Pelotas (UFPEL), com 199 idosos de ambos os sexos - apresentando idade média de 70,03 $( \pm 6,17)$ anos e outro estrato etário de $65( \pm 8,18)$ - investigou os motivos pelos quais idosos aderem a programas de atividade física ${ }^{(13,14)}$. Mais uma vez, pode-se observar que a maioria dos estudos com grupos envolvem pessoas idosas, devido ao aumento dessa população e ao estímulo das políticas públicas que contribuem com estratégias de incentivo ao envelhecimento saudável para prevenção do avanço das doenças crônicas não transmissíveis. Tais estratégias viabilizam e contribuem para que mais pessoas 
alcancem idades avançadas, com o melhor estado de saúde possível $^{(1)}$.

A partir do presente estudo, pode-se inferir que grande parte dos indivíduos envolvidos no grupo de estudo é composta de mulheres, confirmando os dados encontrados na literatura ${ }^{(13-15)}$, o que mostra que as mulheres são mais participativas do que os homens. Corroborando com esses resultados, outro estudo realizado em Pelotas/RS demonstra que apenas 4 homens comportam a amostra, em contraste a 195 mulheres $^{(13)}$. Tal diferença de gênero também aparece nos estudos realizados em Florianópolis/SC: no primeiro, $79 \%$ pertencem ao gênero feminino; no segundo, $91 \%{ }^{(14,15)}$.

Uma pesquisa multicêntrica objetivou investigar como se dá a relação homem/assistência à saúde em cidades de quatro estados brasileiros: Pernambuco (Recife e Olinda), Rio de Janeiro (Rio de Janeiro), Rio Grande do Norte (Natal) e São Paulo (São Paulo e Santos). Foram verificadas as percepções dos homens sobre os serviços de APS e notou-se que eles enxergam esse espaço como feminilizado, o que lhes provocaria a sensação de não pertencimento. Isso aponta para o fato de que os serviços de APS se destinam às pessoas idosas, às mulheres e às crianças, Os homens participam menos de consultas de enfermagem - as quais se orientam, sobretudo, para o acompanhamento do pré-natal e da puericultura - e de atividades educativas. É interessante notar que, mesmo quando se considera a clientela idosa, na qual há quantidade significativa de homens, há pouca presença masculina nos grupos educativos ${ }^{(16)}$.

Sob essa perspectiva, um estudo com 59 homens moradores do município de Coronel Fabriciano/MG evidenciou que a demanda dos homens nos serviços limitase a ações de cunho curativo, a partir de alguma doença já instalada, como diabetes e hipertensão; diferentemente de crianças, mulheres e idosos, que comparecem aos serviços de saúde de forma mais preventiva ${ }^{(17)}$.

Quanto às variáveis relacionadas aos motivos de adesão aos grupos de promoção à saúde de uma ESF, observa-se que os fatores motivacionais dos usuários para participarem dos grupos de promoção da saúde na atenção básica estão relacionados à saúde física, mental e social. Considerase que estes envolvem as relações pessoais, bem como os aspectos psicossociais.

Estudos reforçam que atividades educativas com grupos são um importante dispositivo de promoção da saúde e servem como alternativa para as práticas assistenciais, pois permitem o aprofundamento de discussões e a ampliação de conhecimentos, de modo que as pessoas superem suas dificuldades e obtenham maior autonomia, melhores condições de saúde e qualidade de vida ${ }^{(18-20)}$.

Entre os principais motivos assinalados pelos participantes da pesquisa realizada com 40 mulheres idosas que participavam de programas de promoção da saúde, em Belo Horizonte e Betim, Minas Gerais, estão: melhorar ou manter o estado de saúde $(92,5 \%)$, aumentar o contato social $(85 \%)$, prevenir doenças $(85 \%)$, aprender novas atividades $(82,5 \%)$, e aumento da autoestima $(82,5 \%)^{(21)}$.

Uma pesquisa realizada em Florianópolis analisou os motivos de ingresso de 112 idosos em um programa de atividade física da Universidade do Estado de Santa Catarina e identificou as seguintes variáveis: indicação médica, manter/melhorar a saúde, e necessidade de fazer atividade física (por gosto e sentir-se bem) ${ }^{(22)}$. Vale destacar que tais resultados se assemelham aos obtidos nesta pesquisa.

No que diz respeito aos motivos que levaram os idosos a participar de um projeto de atividade física em Pelotas/ RS, observou-se que a maioria deles busca evitar ou prevenir problemas de saúde $(68,8 \%)$ ou praticar atividade física para seguir orientação médica $(59,8 \%)$. Outros aspectos enfatizados nessa pesquisa realizada em Pelotas contemplam a manutenção da flexibilidade e agilidade $(56,3 \%)$, a ajuda na superação do estresse ou diminuição da tensão $(40,2 \%)^{(13)}$. Interessante assinalar que tais motivos não figuram entre os importantes para os participantes da presente pesquisa.

Neste estudo, as maiores frequências apresentadas como sem importância entre os motivos de adesão encontram-se relacionadas à influência da mídia, em especial dos programas de televisão e das propagandas. $\mathrm{Na}$ pesquisa desenvolvida em Belo Horizonte e Betim, Minas Gerais, os pesquisadores encontraram dados diferentes. As menores frequências associadas aos motivos para a adesão das idosas foram: emagrecer (37,5\%), melhorar a qualidade do sono $(20 \%)$ e reduzir o nível de estresse $(17,5 \%)^{(21)}$.

Os aspectos levantados permitem inferir que os usuários não são tão suscetíveis à influência da mídia no que tange à sua motivação em participar dos programas de saúde oferecidos. No estudo realizado pelo Laboratório de Estudos em Práticas Esportivas e Lazer (LAPEL) da ESEF/ UPE, esses resultados causaram surpresa, pois o poder público contribui na divulgação de políticas públicas de saúde, com programas de promoção da saúde que objetivam incentivar hábitos saudáveis. Portanto, para o autor, a mídia pode influenciar de alguma maneira os indivíduos a praticarem exercícios através da motivação ${ }^{(12)}$. Tais dados e posicionamento estão de acordo com os resultados deste estudo, pois houve essa mesma percepção entre o público analisado.

Quanto às variáveis, no que tange aos motivos de permanência nos grupos de promoção à saúde de uma ESF, observa-se nesse objeto de estudo que todas as categorias analisadas influenciam positivamente a adesão e permanência dos usuários nos grupos de saúde. Isso porque 
os indicadores apontaram que as intervenções têm sido conduzidas com eficiência no que diz respeito ao incentivo a um estilo de vida saudável, com apoio dos profissionais e pessoas envolvidas nas atividades grupais.

Os resultados de um estudo realizado com 30 idosos, de ambos os sexos, no município de Santa Cruz do Sul/ $\mathrm{RS}$, demonstraram que eles se sentem beneficiados com a prática regular de atividade física, bem como se sentem motivados a frequentar assiduamente os grupos. No que se refere às questões relacionadas à motivação, os idosos encontram razões de participação em diferentes aspectos: sociais, físicos e psíquicos. Todos esses aspectos, por sua vez, indicam a busca da qualidade de vida ${ }^{(23)}$. Já no estudo realizado pelo Grupo de Estudos da Terceira Idade, da UDESC, o principal motivo para os idosos permanecerem em um programa de atividade física concerne a sentiremse bem, já que essa prática tende a melhorar a saúde física e mental, além de proporcionar convívio social/amizade/ socialização ${ }^{(22)}$.

No trabalho realizado com 120 idosos de dois programas de exercícios físicos, em Recife/PE, observouse que no item importantíssimo dos diferentes motivos para a permanência nos programas mereceram destaque: manter a saúde $(100 \%)$, melhorar a postura (75\%), sentir bem-estar provocado pelo exercício $(74,2 \%)$, aumentar o bem-estar corporal $(73,3 \%)$, manter-se em forma $(70,8 \%)$, prazer da prática $(66,7 \%)$, ficar mais forte $(62,5 \%)$, receber incentivos do professor $(62,5 \%)$, sentir bem-estar provocado pelo ambiente $(60 \%)$, sentir-se realizado $(57,5 \%)$, receber atenção do professor $(57,5 \%)$, construir amizades $(47,5 \%)$ ${ }^{(12)}$. Vale destacar que os resultados obtidos pela referida pesquisa também encontram respaldo neste estudo.

Verificou-se ainda que os participantes desta pesquisa consideram importantíssimo: a alegria do ambiente, a atenção que recebem dos profissionais (professor) e a construção de amizades. Os estudos desenvolvidos no Sul do Brasil assinalam que os participantes encontram nos grupos formas de estabelecer e estreitar laços de amizade e solidariedade ${ }^{(24,25)}$ Destaca-se o papel fundamental da socialização obtida no trabalho em grupo.

Uma entrevista realizada com idosos de Ribeirão Preto/SP identificou que os grupos de promoção à saúde possibilitaram, além de distração e lazer, trocas de experiências e interações. Estas transformaram de modo significativo suas relações sociais, na medida em que os encontros grupais possibilitaram aos participantes novos relacionamentos e a ampliação da rede social de apoio, além de uma percepção de melhora na saúde e, consequentemente, na qualidade de $\operatorname{vida}^{(26,27)}$.

Nota-se a importância da atenção recebida pelas pessoas por parte dos profissionais e o estímulo a adesão e permanência nos grupos. Dessa forma, o trabalho em grupos favorece o vínculo entre o profissional da saúde e a pessoa, independentemente de ser idosa ou não, sendo um espaço complementar à consulta individual para troca de informações, orientação e educação em saúde ${ }^{(27)}$.

Um elemento citado como sem importância entre os motivos de permanência nos grupos de promoção da saúde foi o status social (11\%), aproximando-se do trabalho, em que os participantes de programas de atividade física consideraram como sem importância os fatores relacionados a ter status social (35\%), atenção dos funcionários do local (18,3\%), incentivo da família e dos amigos (17,5\%), emagrecer $(17,5 \%)$ e parecer mais jovem $(11,7 \%)^{(12)}$.

É importante frisar que não se realizou um cálculo amostral para a área de abrangência da UESF Lídia, uma vez que a pesquisa desenvolvida deu-se com um grupo de estudo. Assim, o número de sujeitos ficou limitado. Por conseguinte, deixa-se como sugestão para que novas pesquisas abordem uma análise qualitativa dos motivos de adesão e permanência nos grupos, considerando a satisfação dos usuários em relação ao convívio social dos grupos.

\section{CONCLUSÃO}

Diante dos dados observados, com relação aos motivos de adesão aos grupos, os indivíduos tendem a buscar melhores condições de saúde e de qualidade de vida, ao passo que o incentivo a um estilo de vida mais saudável, como também o apoio dos profissionais e demais pessoas envolvidas nas atividades, implica em maior permanência dos usuários no grupo.

\section{REFERÊNCIAS}

1. Ministério da Saúde (BR), Secretaria de Atenção à Saúde, Departamento de Ações Programáticas e Estratégicas. Atenção à saúde da pessoa idosa e envelhecimento. Brasília: Ministério da Saúde; 2010.

2. Araújo JD. Polarização epidemiológica no Brasil. Epidemiol Serv Saúde. 2012;21(4):533-8.

3. Conselho Nacional de Secretários de Saúde (BR). Atenção primária e promoção da saúde. Brasília: CONASS; 2011.

4. More CLOO, Ribeiro C. Trabalhando com grupos na estratégia saúde da família. Florianópolis: UNASUS; 2010 [acesso em 2016 Fev 11]. Disponível em: https:// ares.unasus.gov.br/acervo/handle/ARES/195

5. Brasil. Portaria n 2.488 de 21 de outubro de 2011: Política Nacional de Atenção Básica. [acesso em 2012 maio 28]. Disponível em: http://bvsms.saude.gov.br/ bvs/saudelegis/gm/2011/prt2488_21_10_2011.html 
6. Costa JA, Balga RSM, Alfenas RCG, Cotta RMM. Promoção da saúde e diabetes: discutindo a adesão e a motivação de indivíduos diabéticos participantes de programas de saúde. Ciênc \& Saúde Coletiva, 2011;16(3):2001-9.

7. Magnabosco P, Nogueira MS. Avaliação da contribuição do grupo de convivência para o cuidado do indivíduo hipertenso. Rev Eletrônica Enferm [Internet]. 2011 [acesso em 2016 fev 13];13(1):110-7. Disponível em: http://www.fen.ufg.br/revista/v13/n1/v13n1a12.htm.

8. Bittar C, Lima LCV. O impacto das atividades em grupo como estratégia de promoção da saúde na senescência. Rev Kairós. 2011;14(4):101-18.

9. Lakatos EM, Marconi MA. Metodologia científica. São Paulo: Atlas; 1986.

10. Minayo MCS. O desafio da pesquisa social. $27^{\mathrm{a}}$ ed. Petrópolis: Vozes; 2008.

11. Gil AC. Como elaborar projetos de pesquisa. $5^{\mathrm{a}}$ ed. São Paulo: Atlas; 2008.

12. Freitas CMSM, Santiago MS, Viana AT, Leão AC, Freyre C. Aspectos motivacionais que influenciam a adesão e manutenção de idosos a programas de exercícios físicos. Rev Bras Cineantropom Desempenho Hum. 2007;9(1):92-100.

13. Ribeiro JAB et al.. Adesão de idosos a Programas de atividade física: motivação e significância. Rev Bras Ciênc Esporte. 2012;34(4):969-84.

14. Meuer ST, Benedetti TRB, Mazo GZ. Motivação de idosos para a adesão a um programa de exercícios físicos. Psicol Am Lat. 2009 [acesso em 2015 Jan 26]:18. Disponível em: http://psicolatina.org/18/ motivacion.html

15. Benedetti TR, Mazo GZ, Borges LJ. Condições de saúde e nível de atividade física em idosos participantes e não participantes de grupos de convivência de Florianópolis. Ciênc Saúde Coletiva. 2012;17(8):208793.

16. Couto MT, Pinheiro TF, Valença O, Machin R, Silva GSN, Gomes R et al. O homem na atenção primária à saúde: discutindo (in)visibilidade a partir da perspectiva de gênero. Interface Comun Saúde Educ. 2010;14(33):257-70.

17. Albano BR, Basílio MC, Neves JB. Desafios para a inclusão dos homens nos serviços de Atenção Primária à Saúde. Rev Enfermagem Integrada. 2010;3(2): 554-63.
18. Rumor PCF, Berns I, Heideman ITSB, Mattos LHL, WosnyAM. A promoção da saúde nas práticas educativas da saúde da família. Cogitare Enferm. 2010;15(4):674-80.

19. Araújo LF, Coelho CG, Mendonça ET, Vaz AVM, Siqueira-Batista R, Cotta RMM. Evidências da contribuição dos programas de assistência ao idoso na promoção do envelhecimento saudável no Brasil. Rev Panam Salud Publica. 2011;30(1):80-6.

20. Alves GG, Aerts D. As práticas educativas em saúde e a Estratégia Saúde da Família. Ciênc Saúde Coletiva. 2011;16(1):319-25.

21. Gomes KT, Zazá DC. Motivos de adesão a prática de atividade física em idosas. Rev Bras Ativ Fís Saúde. 2009;14(2):132-8.

22. Conceição JCR, Krug RR, Gonçalves E, Mazo GZ. Motivos de adesão e permanência em um programa de atividade física para idosos. EFDeportes.com [Internet]. 2011[acesso em 2015 Jan 26];16(156). Disponível em: http://www.efdeportes.com/efd159/ motivos-de-adesao-e-permanencia-de-idosos.htm

23. Garske Q, Swanke NL, Reuter EM, Morais GG, Weis GF. Motivação e barreiras: percepção de idosos que participam de grupos de terceira idade de Santa Cruz do Sul. Cinergis. 2011;12(1):48-54.

24. Wichmann FMA, Couto AN, Areosa SVC, Montanes MCM. Grupos de convivência como suporte ao idoso na melhoria da saúde. Rev Bras Geriatr Gerontol. 2013;16(4):821-32.

25. Ferreira-Neto JL, Kind L. Práticas Grupais como dispositivo na promoção de saúde. Physis (Rio J). 2010;20(4):1119-42.

26. Tahan J, Carvalho ACD. Reflexões de Idosos Participantes de grupos de promoção de saúde acerca do envelhecimento e da qualidade de vida. Saúde Soc. 2010;19(4):878-88.

27. Ministério da Saúde (BR), Secretaria de Atenção à Saúde, Departamento de Atenção Básica. Núcleo de apoio à saúde da família: ferramentas para a gestão e para o trabalho cotidiano. Brasília: Ministério da Saúde; 2014. v. 1. (Cadernos de Atenção Básica, n. 39)

\section{Endereço para correspondência:}

Caroline Silva de Freitas

Universidade Federal de Santa Maria - UFSM

Av. Roraima, 1000

Bairro: Camobi

CEP: 97105-900 - Santa Maria - RS - Brasil

E-mail: carolfisio.88@gmail.com 